\title{
Lack of prolactin receptor signaling in mice results in lactotroph proliferation and prolactinomas by dopamine-dependent and -independent mechanisms
}

\author{
Kathryn G. Schuff, ${ }^{1}$ Shane T. Hentges, ${ }^{1}$ Michele A. Kelly, ${ }^{1}$ Nadine Binart, ${ }^{2}$ Paul A. Kelly, \\ P. Michael Iuvone, ${ }^{3}$ Sylvia L. Asa, ${ }^{4}$ and Malcolm J. Low ${ }^{1,5}$ \\ ${ }^{1}$ Vollum Institute, Oregon Health \& Science University, Portland, Oregon, USA \\ ${ }^{2}$ Institut National de la Santé et de la Recherche Médicale (INSERM), Faculté de Necker, Paris, France \\ ${ }^{3}$ Department of Pharmacology, Emory University School of Medicine, Atlanta, Georgia, USA \\ ${ }^{4}$ Department of Pathology, University Health Network, Toronto, Ontario, Canada \\ ${ }^{5}$ Department of Behavioral Neuroscience, Oregon Health \& Science University, Portland, Oregon, USA
}

\begin{abstract}
Hypothalamic dopamine inhibits pituitary prolactin secretion and proliferation of prolactin-producing lactotroph cells by activating lactotroph dopamine D2 receptors (D2Rs). Conversely, prolactin (PRL) stimulates hypothalamic dopamine neurons via PRL receptors (PRLRs) in a short-loop feedback circuit. We used $\mathrm{Drd2}^{-/-}$and $\mathrm{Prlr}^{-/-}$mutant mice to bypass this feedback and investigate possible dopamine-independent effects of PRL on lactotroph function. The absence of either receptor induced hyperprolactinemia and large prolactinomas in females. Small macroadenomas developed in aged $\mathrm{Prlr}^{-/}$males, but only microscopic adenomas were found in $\mathrm{Drd2}^{-/-}$male mice. Pharmacologic studies in $\mathrm{Prlr}^{-/}$mice with D2R agonists and antagonists demonstrated a significant loss of endogenous dopamine tone, i.e., constitutive inhibitory signaling by the D2R, in the pituitary. However, $\mathrm{Prlr}^{-1-}$ mice exhibited more profound hyperprolactinemia and larger tumors than did age-matched $\operatorname{Drd2^{-/-}}$ mice, and there were additive effects in compound homozygous mutant male mice. In vitro, PRL treatment markedly inhibited the proliferation of wild-type female and male $\operatorname{Drd2^{-/-}}$ lactotrophs, but had no effect on female $\operatorname{Drd2^{-/-}}$ lactotrophs, suggesting a downregulation or desensitization of PRLR in response to chronic hyperprolactinemia. We conclude that PRL inhibits lactotrophs by two distinct mechanisms: (a) indirectly by activation of hypothalamic dopamine neurons and (b) directly within the pituitary in a dopamine-independent fashion.
\end{abstract}

J. Clin. Invest. 110:973-981 (2002). doi:10.1172/JCI200215912.

\section{Introduction}

Human lactotroph adenomas (prolactinomas) are the most frequent functioning pituitary tumors (1), but their pathogenesis remains elusive. The primary regulation of prolactin (PRL) secretion is mediated by the inhibitory effects of dopamine released from the median eminence of the hypothalamus and acting at the dopamine $\mathrm{D} 2$ receptor subtype (D2R) (2). Mice deficient in the D2R receptor ( $\mathrm{Drd2} 2^{-/-}$mice) have previously been shown to develop hyperprolactinemia, lactotroph hyperplasia, and prolactinomas $(3,4)$, confirming a crit-

Received for publication May 10, 2002, and accepted in revised form August 7, 2002.

Address correspondence to: Malcolm Low, Vollum Institute L-474, Oregon Health \& Science University, 3181 SW Sam Jackson Park Road, Portland, Oregon 97201-3098, USA.

Phone: (503) 494-4672; Fax: (503) 494-4976;

E-mail: low@OHSU.edu.

Kathryn G. Schuff and Shane T. Hentges contributed equally to this work.

Conflict of interest: No conflict of interest has been declared. Nonstandard abbreviations used: prolactin (PRL); dopamine D2 receptor (D2R); tyrosine hydroxylase (TH); PRL receptor (PRLR); growth hormone $(\mathrm{GH})$; thyroid-stimulating hormone (TSH); follicle-stimulating hormone (FSH); luteinizing hormone (LH); adrenocorticotropic hormone (ACTH); 3,4-dihydroxyphenylalanine (DOPA); aromatic amino acid decarboxylase (AADC). ical role of hypothalamic dopamine and dopamine receptor activation in the physiologic regulation of lactotroph proliferation and PRL secretion. However, the involvement of a number of other regulatory factors has been postulated, including PRL itself (5). PRL increases hypothalamic-pituitary dopamine tone, i.e., constitutive inhibitory signaling by the D2R, and decreases PRL secretion (6-8) by altering the expression (9) and activity (7) of tyrosine hydroxylase (TH), the rate-limiting enzyme in dopamine synthesis.

In addition to its indirect effects, mediated by changes in hypothalamic dopamine, we hypothesize that PRL might have direct effects on the pituitary gland. PRL receptors (PRLRs) have been demonstrated in the anterior pituitary in several species, including mouse $(10,11)$, and have been found specifically on lactotrophs in rats (12) and humans (13). Its effects on proliferation of other cell types are varied, but are predominantly stimulatory (14-25) except in the ovary $(26,27)$, adrenal gland $(28)$, and vascular endothelium (29). Although it has been postulated that PRL may play an autocrine or paracrine role in lactotroph proliferation (11), there is limited empirical evidence supporting this theory. Experiments to evaluate a direct pituitary effect of PRL in vivo are difficult because of its concurrent effects on hypothalamic dopamine. By 
crossing PRLR-deficient (Prlr/-) mice with $\mathrm{Drd2}^{-/-}$ mice, we were able to evaluate a possible contribution of dopamine-independent effects of PRL signaling on the control of PRL secretion and lactotroph growth.

\section{Methods}

Generation of receptor-deficient mice. Generation of $\mathrm{Drd} 2^{-/}$and $\mathrm{Prlr}^{-/}$mice by gene targeting in embryonic stem cells has been previously described $(3,30)$. Each receptor-deficient line of mice was independently backcrossed to inbred C57BL/6J mice for five or more generations to produce incipient congenic lines and reduce variability from gene epistasis. Because of impaired fertility, lactation $(30,31)$, and maternal behavior (32) in $\mathrm{Prlr}^{-/}$mice, a selective breeding strategy and inclusion of CD-1 foster mothers were used in the intercrosses to produce sibling cohorts of wildtype, $\mathrm{Drd2} 2^{--}$, $\mathrm{Prlr}^{-1-}$, and compound mutant ( $\mathrm{Drd2} 2^{-/}$, $\left.\mathrm{Prlr}^{-/-}\right)$mice for these studies. All animal care practices and experimental procedures were approved by the Oregon Health \& Science University Institutional Animal Care and Use Committee.

Determination of serum PRL. Serum was obtained under brief isoflurane anesthesia from a tail bleed and frozen at $-80^{\circ} \mathrm{C}$ until analysis. PRL radioimmunoassay used mouse PRL (mPRL) reference preparation AFP6476C, mPRL AFP1077D for iodination, and antimPRL antiserum AFP131078 (provided by A.F. Parlow and the National Hormone and Peptide Program, Harbor-UCLA Medical Center, Torrance, California, USA). The assay was performed as directed, except that mPRL was iodinated with $3 \mu \mathrm{g}$ chloramine-T (Sigma-Aldrich, St. Louis, Missouri, USA) per $\mu \mathrm{g}$ peptide and purified with Dowex 20-50 chloride mesh resin beads (Bio-Rad Laboratories Inc., Hercules, California, USA); approximately 20,000 cpm was added to each tube. Assay sensitivity was $15 \mathrm{ng} / \mathrm{ml}$ using $10-\mu \mathrm{l}$ serum samples. Samples were analyzed in duplicate at multiple dilutions. For drug responses, basal serum was collected from 15month-old male mice, then $5 \mathrm{mg} / \mathrm{kg}$ 2-bromo- $\alpha$ ergocryptine (bromocriptine; Sigma-Aldrich) or 10 $\mathrm{mg} / \mathrm{kg}$ haloperidol (Ortho-McNeil Pharmaceutical, Raritan, New Jersey, USA) was administered intraperitoneally, and post-treatment serum samples were obtained 1 hour later.

Histologic analyses. Mice were sacrificed by decapitation at ages $6,12,14$, or $18-21$ months, and their pituitary glands were inspected visually with a dissecting microscope, weighed, and placed immediately in $10 \%$ buffered formalin. Paraffin-embedded sections 4-5 $\mu \mathrm{m}$ thick were stained with hematoxylin and eosin or the Gordon-Sweet silver method for reticulin matrix. Immunohistochemical staining to identify adenohypophyseal hormones was performed using the streptavidin-biotin peroxidase technique. Primary antisera directed against rat pituitary hormones were used at the following dilutions: growth hormone $(\mathrm{GH})$, 1:2,500; PRL, 1:2,500; thyroid-stimulating hormone (TSH- $\beta$ ), 1:3,000; follicle-stimulating hormone- $\beta$
(FSH- $\beta$ ), 1:600; luteinizing hormone- $\beta$ (LH- $\beta$ ), $1: 2,500$ (all provided by A.F. Parlow and the National Hormone and Peptide Program); and prediluted adrenocorticotropic hormone (ACTH), further diluted 1:20 (DAKO Corp., Carpinteria, California, USA).

Evaluation of hypothalamic dopaminergic neuron function. TH gene expression was evaluated by in situ hybridization. Mice were sacrificed by decapitation, and brains were removed and snap-frozen in isopentane over dry ice and stored at $-80^{\circ} \mathrm{C}$. Ten-micrometer sections were cut on a cryostat and thaw-mounted onto gelatin-coated slides. TH mRNA was detected by hybridization at $60^{\circ} \mathrm{C}$ with 20 million $\mathrm{cpm} / \mathrm{ml}$ of a ${ }^{35} \mathrm{~S}$-UTP-labeled antisense riboprobe (provided by E. Lewis, Department of Biochemistry and Molecular Biology, Oregon Health \& Science University, Portland, Oregon, USA). The slides were exposed to NBT2 emulsion (Eastman Kodak, Rochester, New York, USA) for 15 days, developed, and counterstained with neutral red. Digital images were captured using both bright- and dark-field illumination on a Leica research microscope (Leica Microsystems Inc., Deerfield, Illinois, USA). TH activity was estimated by the measurement of 3,4-dihydroxyphenylalanine (DOPA) accumulation after inhibition of aromatic amino acid decarboxylase (AADC) by m-hydroxybenzylhydrazine dihydrochloride (NSD 1015; Sigma-Aldrich). Briefly, mice were sacrificed by decapitation 30 minutes after an intraperitoneal injection of $100 \mathrm{mg} / \mathrm{kg} \mathrm{NSD} \mathrm{1015.} \mathrm{Whole} \mathrm{hypothalamic}$ blocks and a portion of dorsal striatum were snapfrozen in isopentane on dry ice and stored at $-80^{\circ} \mathrm{C}$ until analyzed. Hypothalami were sonicated in 20 volumes and striata in 50 volumes of homogenization solution and subjected to HPLC and electrochemical detection of DOPA as previously described (33).

Primary cultures of anterior pituitary cells. Anterior pituitary glands were collected from randomly cycling C57BL/6J mice and placed into ice-cold HBSS (Sigma-Aldrich) and dissociated enzymatically in HBSS containing collagenase, deoxyribonuclease, and BSA as described previously (34). Cells were plated at 250,000 cells/well on poly-L-lysine-coated glass coverslips in 24-well plates in DMEM/Ham's F12 medium (1:1; Sigma-Aldrich) containing $50 \mathrm{U} / \mathrm{ml}$ penicillin $\mathrm{G}, 50 \mu \mathrm{g} / \mathrm{ml}$ streptomycin, and $10 \% \mathrm{FBS}$ for 24 hours. The next day, 24 hours prior to PRL treatment, the medium was replaced with DMEM/F12 supplemented with Serum Replacement 2 (SigmaAldrich). For the 48-hour PRL treatment, the medium was replaced with DMEM/F12/Serum Replacement 2 containing recombinant mPRL $(0-10 \mu \mathrm{g} / \mathrm{ml}$; provided by A.F. Parlow and the National Hormone and Peptide Program). The media and treatment were changed at 24 hours, and $0.1 \mathrm{mM}$ bromodeoxyuridine (BrdU; Sigma-Aldrich) was added for the last 12 hours of PRL treatment.

Lactotroph cell proliferation. Lactotroph cell proliferation was determined by identifying the cells that displayed immunoreactivity for both BrdU and PRL. After 
treatment with mPRL and BrdU as above, cells were washed and fixed in 99\% ice-cold ethanol and processed for immunofluorescence. The mouse monoclonal BrdU antibody (1:200; Becton Dickinson Immunocytometry Systems, San Jose, California, USA) and rabbit polyclonal mouse-specific PRL antibody (1:3,000; gift of F. Talamantes, Biology, University of California at Santa Cruz, Santa Cruz, California, USA) were detected with goat anti-mouse FITC-conjugated secondary antibodies (1:200; BioSource International, Camarillo, California, USA) and goat anti-rabbit rhodamine-conjugated secondary antibodies (1:200; American Qualex, San Clemente, California, USA). Cell nuclei were fluorescently labeled with Hoechst 33258 (Sigma-Aldrich). Cells immunoreactive for both BrdU and PRL were counted as dividing lactotrophs. Cell counts were made at $20 \times$ magnification on a Leica epifluorescence microscope. An investigator blind to the treatment and genotype counted five random areas in each coverslip, approximately 250 cells/area. Six to eight coverslips per treatment from three separate cultures were evaluated for each genotype.

Statistical analyses. Statistical analyses were performed using StatView statistical analysis software (SAS Institute Inc., Cary, North Carolina, USA). For comparisons within a genotype before and after drug treatment, onetailed paired Student $t$ tests were used because of the well-described direction of change. For comparisons among multiple genotypes, ANOVA was used. Post hoc analyses were by the Fisher post hoc test. All data are presented as mean \pm SEM. $P$ values less than 0.05 were considered significant.

\section{Results}

Hyperprolactinemia and pituitary pathology. Serum PRL increased exponentially with age in $\mathrm{Drd2} 2^{-/-}$mice, $\mathrm{Prlr}^{-/}$ mice, and compound receptor-deficient $\left(\mathrm{Drd} 2^{-/}\right.$, Prlr ${ }^{-1}$ ) mice (Figure 1, a and b). Levels were more markedly elevated in mutant females than males at all timepoints. In addition, there was earlier and more severe hyperprolactinemia in both sexes of $\mathrm{Prlr}^{-/-}$mice than in $D r d 2^{-/-}$mice, and additive effects of the combined mutations were apparent at both 12 and 18 months of age in males (Figure 1b).

Similar to our previous observations in $\mathrm{Drd2} 2^{-/-}$mice $(3,4)$, pituitary glands in $\mathrm{Prlr}^{-/}$mice were significantly enlarged compared with those in the wild type starting at 6 months, and continued to increase in size in the older groups of female and male mice (Figure 1, $c$ and d). However, at 6 months, the female $\mathrm{Prlr}^{-/}$pituitary glands already exhibited distinct lactotroph hyperplasia (data not shown), a histological feature delayed until 10-12 months in female Drd2 $2^{--}$mice (3). The pituitary glands of 14-month-old female $\mathrm{Prlr}^{-/}$and compound receptor-mutant mice contained massive, multifocal tumors histologically similar to, though significantly larger than, those observed in female $\operatorname{Drd2^{-/}}$ mice of the same age. Histopathology of these tumors demonstrated that they were all monohormonal lactotroph adenomas characterized by extensive breakdown of the reticulin network, peliosis, and enlarged, pleomorphic nuclei with numerous mitotic figures (Figure 2). Hypertrophied perinuclear Golgi networks were observed, indicating a hypersecretory state similar to that observed in human prolactinomas. Immunostaining
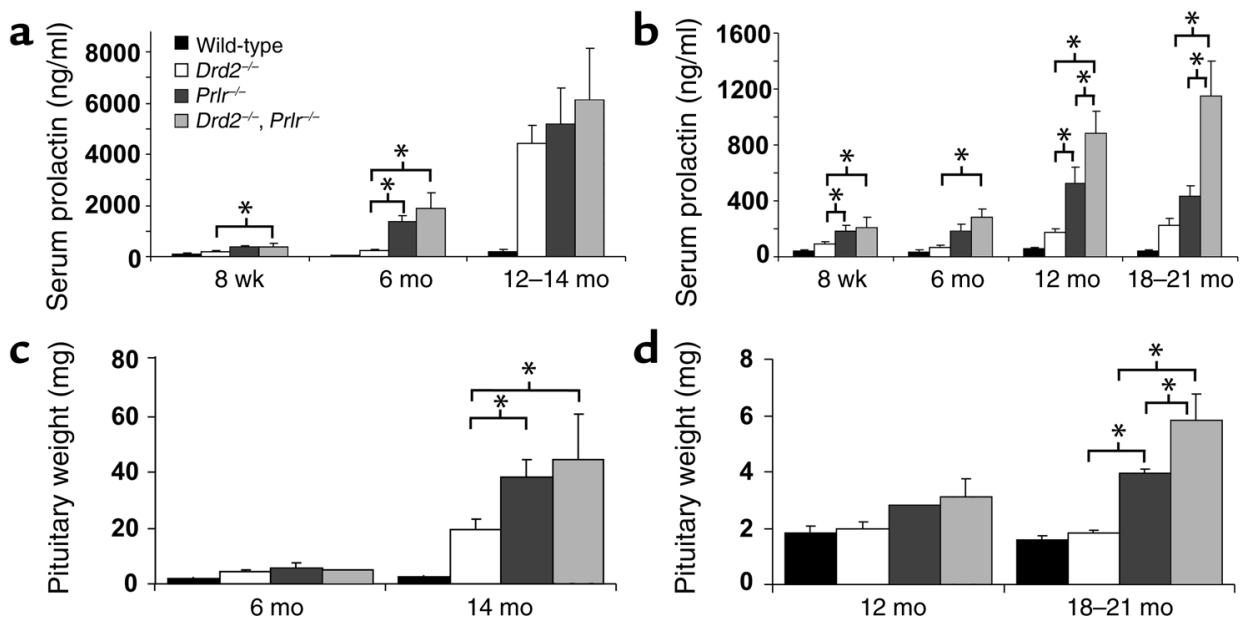

\section{Figure 1}

Hyperprolactinemia and pituitary enlargement in $\mathrm{Drd} 2^{-/-}$and $\mathrm{Prl}{ }^{-1-}$ mice. Serum PRL increased with age in both female (a) and male (b) Drd2 ${ }^{-1-}$ and $\mathrm{PrIr}^{-1-}$ mice and was more marked in Prlr ${ }^{-1}$ mice. Deficiency of both receptors produced an additive effect on hyperprolactinemia in male mice. Prlr ${ }^{-1}$ mice of both sexes developed hyperprolactinemia at younger ages than did Drd2 $2^{-1-}$ mice. $P<0.005$ for ANOVA of main effect of genotype at all timepoints. ${ }^{*} P<0.05$ for pairwise comparisons by the Fisher post hoc test. $n=6-22$ for ages 8 weeks and 6 months, and $n=4-8$ for ages 12 months and older. (c) Female $\mathrm{Drd} 2^{-/-}$mice and $\mathrm{Prlr}^{-1-}$ mice had slightly enlarged pituitary glands at 6 months and massively enlarged glands at 14 months (10- to 20-fold greater than wild-type). (d) Moderate pituitary enlargement occurred only at 18-21 months in $\mathrm{PrIr}^{-1-}$ and compound homozygous mutant male mice. $P<0.001$ for ANOVA of main effect of genotype for females at 14 months and males at 18-21 months. ${ }^{*} P<0.05$ for pairwise comparisons using the Fisher post hoc test; $n=3-4$ for 6 -month-old females and 12 -month-old males (except $n=1$ for Prlr ${ }^{-1}$ male and $n=2$ for Drd2-/- males); $n=5-7$ for 14-month-old females and 18- to 21-month-old males. 

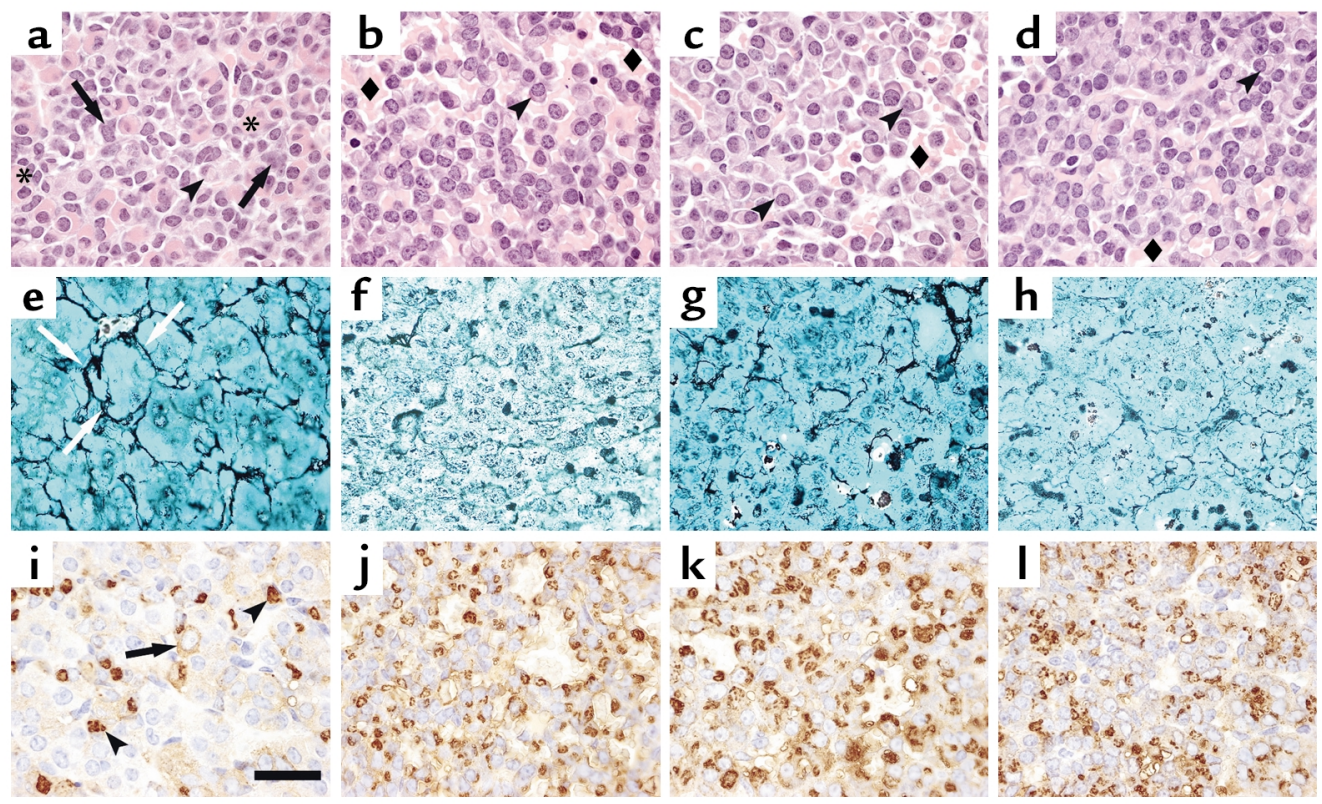

\section{Figure 2}

Histological analysis of anterior pituitaries from 14-month-old female mice. Top row shows hematoxylin and eosin-stained pituitary sections of wild-type (a), $\operatorname{Drd} 2^{-/-}(\mathbf{b}), \operatorname{Prlr}^{--}(\mathbf{c})$, and compound mutant $\left(\operatorname{Drd}^{-/-}, \operatorname{Prl} r^{\prime-}\right)(\mathbf{d})$ mice. The wild-type pituitary has a normal architecture and mixture of cells including large acidophilic somatotrophs (asterisks), basophils with juxtanuclear, complex lysosomal bodies (arrows), and scattered chromophobic lactotrophs with clear, juxtanuclear Golgi regions (arrowheads). The three mutant genotypes (b-d) all exhibit peliosis (extravasated erythrocytes not contained in capillaries; diamonds) and hypertrophic lactotrophs with very large Golgi regions (arrowheads) and scattered, large, hyperchromatic, atypical nuclei. Middle row: Gordon-Sweet silver stain shows the normal acinar pattern and reticulin network (arrows) in the wild-type pituitary (e), but reveals significant disruption of the reticulin network in $\operatorname{Drd} 2^{-/-}$mice (f) and expanded acini and partial loss of reticulin in the $\mathrm{Prlr}^{--}(\mathbf{g})$ and compound mutant $\left(\operatorname{Drd} 2^{-/-}, \mathrm{PrIr}^{--}\right)$(h) mice, findings that are pathognomonic for adenomatous transformation of pituitary cells. Bottom row: PRL immunohistochemistry. The wild-type pituitary (i) contains many mammosomatotrophs with pale, diffuse immunostaining (arrow) and a few mature active lactotrophs with PRL immunostaining in the Golgi apparatus (arrowheads). Almost all the cells in the $\operatorname{Drd}^{-/-}(\mathbf{j})$ and $\mathrm{Prlr}^{-/-}(\mathbf{k})$ tumors are active lactotrophs with PRL immunoreactivity in the Golgi. The compound mutant $\left(\mathrm{Drd} 2^{-/-}, \mathrm{Prlr}^{-/}\right)$(I) tumor is similar, but has fewer immunopositive cells than either single mutant. Representative fields of adenomas from each genotype are shown. Scale bar represents $20 \mu \mathrm{m}$; original magnification: $\times 100$, oil immersion.

for GH, LH- $\beta$, ESH $\beta$, TSH- $\beta$, and ACTH was negative in the adenomas (data not shown). Male $\mathrm{Prlr}^{-/}$and compound receptor-deficient $\left(\operatorname{Drd2}^{-/-}, \mathrm{Prlr}^{-/}\right)$mice developed smaller macroadenomas at 18-21 months, with an additive effect in mice with the compound mutation (Figure 1d). Similar histological characteristics were observed in these tumors (data not shown).

Pharmacologic evaluation of bypothalamic dopamine. We evaluated and compared the effect of the loss of PRL or dopamine signaling on hypothalamic dopamine neuron function in $\mathrm{Drd2}^{-/-}$and $\mathrm{Prlr}^{-/}$mice and compound receptor-deficient $\left(\mathrm{Drd2}^{-/-}, \mathrm{Prlr}^{-/-}\right)$mice by three approaches: pharmacologic challenge with a D2R agonist and antagonist followed by measurement of serum PRL; in situ hybridization of mRNA for TH (the ratelimiting enzyme in the synthesis of dopamine); and assessment of TH activity by the quantitation of DOPA after inhibition of AADC. Only male mice were used for these experiments because of the early tumor development observed in female receptor mutant mice and consequent secondary distortion of the overlying stalk/median eminence and basal hypothalamus.

Wild-type male mice had low basal levels of serum PRL, which demonstrated a nonsignificant trend to reduction by the D2R agonist bromocriptine (Figure 3a), but serum PRL levels were significantly increased by the D2R antagonist haloperidol (Figure 3b), illustrating the dopamine inhibitory tone normally present on lactotrophs. $\operatorname{Drd} 2^{-/-}$and compound mutant (Drd2-/, $\left.\mathrm{Prlr}^{-1}\right)$ mice exhibited no response to either drug, as expected from the absence of D2R. In contrast, $\mathrm{Prlr}^{-1}$ mice demonstrated an average $85 \%$ decline in serum PRL 1 hour after bromocriptine treatment (Figure 3a) and no response to haloperidol (Figure $3 \mathrm{~b}$ ), indicating the presence of functional D2R on lactotrophs and low endogenous dopamine tone in the pituitary of these mice.

Hypothalamic TH expression. The loss of dopamine tone in the pituitaries of $\mathrm{Prlr}^{-1-}$ mice could be due to developmental reductions in the number of hypothalamic dopaminergic neurons (35) or decreased function of the neurons. We therefore performed in situ hybridization to detect TH mRNA in hypothalamic neuronal soma of male mice. Compared with levels in wild-type mice (Figure 4, a and d), levels of TH mRNA were qualitatively increased in the arcuate nucleus of $\mathrm{Drd2} 2^{-/-}$mice (Figure $4, \mathrm{~b}$ and e). In contrast, arcuate nucleus TH mRNA levels appeared decreased but clearly still evident in $\mathrm{Prlr}^{-1}$ mice (Figure 4, c and f). TH mRNA levels in dopamin- 
ergic neurons not regulated by PRL, such as the zona incerta (data not shown) and ventral tegmental area/substantia nigra, were comparable in $D r d 2^{-/-}$mice, $\mathrm{Prlr}^{-1-}$ mice, and wild-type mice (Figure 4, g-i).

Hypothalamic dopamine synthesis. To estimate changes in the rate of dopamine synthesis resulting from decreased levels of $\mathrm{TH}$ or decreased activity of $\mathrm{TH}$, DOPA content of brain tissues was measured after blockade of AADC. DOPA levels were significantly higher in striata from $\mathrm{Drd2} 2^{-/-}$mice than in striata from wild-type mice (Figure 4k), but were not significantly higher in the whole hypothalamus (Figure 4j) of Drd2-/mice (although there was a trend toward an increase). In contrast, DOPA accumulation in the striatum and hypothalamus of $\mathrm{Prlr}^{-/}$mice was not significantly different from that in the wild type.

Effects of in vitro PRL treatment. Despite the pharmacologic and neurochemical evidence of reduced tuberohypophyseal dopamine tone in $\mathrm{Prlr}^{-/-}$mice, the phenotypic differences between $\mathrm{Prlr}^{-/-}$and $\mathrm{Drd2} 2^{-{ }_{-}}$mice and additive effects of the combined mutations in males on serum PRL levels and pituitary size suggest that PRL and PRLR have additional dopamine-independent effects on lactotroph function. To determine whether there are direct pituitary effects of PRL on lactotroph growth, we assessed lactotroph proliferation in vitro by culturing dispersed pituitary cells from 10- to 12 month-old wild-type and $D r d 2^{-/-}$mice in the presence of recombinant $\mathrm{mPRL}$ in doses of $0.1-10 \mu \mathrm{g} / \mathrm{ml}$ (Figure 5). Absolute numbers of proliferating lactotrophs from wild-type males were too low to reliably quantitate. PRL treatment reduced the number of PRL-immunoreactive lactotrophs incorporating BrdU by $75 \%$ in pituitary cultures from wild-type female mice (Figure 5a). In contrast, there was no reduction in the number of proliferating lactotrophs in cultures of pituitaries from Drd2-/- female mice (Figure $5 \mathrm{~b}$ ), which in vivo already demonstrate marked hyperprolactinemia, lactotroph hyperplasia, and adenomas at this age. An in vivo BrdU labeling experiment performed in 6-month-old mice (data not shown) revealed a $5 \%$ labeling index of lactotrophs from $\mathrm{Drd} 2^{-/-}$females, similar to the in vitro results; the figure was only $0.75 \%$ in wild-type females, indicating that the much higher basal index of wildtype lactotrophs in primary culture conditions is likely due to the acute loss of dopamine inhibition (2). Unlike the response in female $\operatorname{Drd2^{-/-}}$ mice, PRL reduced the number of proliferating lactotrophs by $73 \%$ in cultures from male $\mathrm{Drd2} 2^{-/-}$mice (Figure 5c), which have only very modest hyperprolactinemia compared with the females $(3,4)$.

\section{Discussion}

Although the finding of hyperprolactinemia in $\mathrm{Prlr}^{-/}$ mice could be predicted based on the known feedback loop of PRL on hypothalamic dopaminergic neurons, the finding of pituitary hyperplasia and adenoma formation was a surprise. Previously, both we (3) and another group (11) concluded from our original stud- ies of $\operatorname{Drd2^{-/-}}$ mice that PRL most likely functioned as a mitogen for lactotrophs. In the majority of tissues where it has been evaluated, PRL is mitogenic (14-25), with only a few tissues demonstrating an antiproliferative role (26-29). However, we show here that in $\mathrm{Prlr}^{-/}$ mice, there are two factors contributing to the release of the lactotroph from its usual secretory and proliferative controls: a decrease in the normally inhibitory dopaminergic control and a second, direct effect at the level of the pituitary that is most consistent with an antiproliferative action of PRL on lactotrophs.

We have demonstrated that a lack of PRLR signaling results in decreased hypothalamic dopamine tone. This was shown pharmacologically by the absence of antagonizable dopamine action in male mice. The finding of an acute decline in serum PRL after bromocriptine treatment demonstrated that D2R signaling was intact in $\mathrm{Prlr}^{-1-}$ mice, but the complete lack of response to the antagonist haloperidol suggests that dopaminergic input to the pituitary gland was very low, despite the marked hyperprolactinemia in these mice.

There are several possible, and not mutually exclusive, mechanisms that must be considered to explain the low levels of tuberoinfundibular dopamine in the $\mathrm{Prlr}^{-1-}$ mice. These include the loss of the hypothalamic neuronal populations responsible for dopaminergic input to the pituitary, decreased levels or activity of TH (the rate-limiting enzyme in dopamine synthesis), and decreased neuronal activity of these hypothalamic neurons. Experiments with Snell dwarf mice indicated a requirement for PRL early in development to promote the normal survival and differentiation of the tuberoinfundibular dopaminergic neurons (35). However, subsequent studies of PRL peptide-deficient mice indicated that a lack of PRL predominantly affected the function
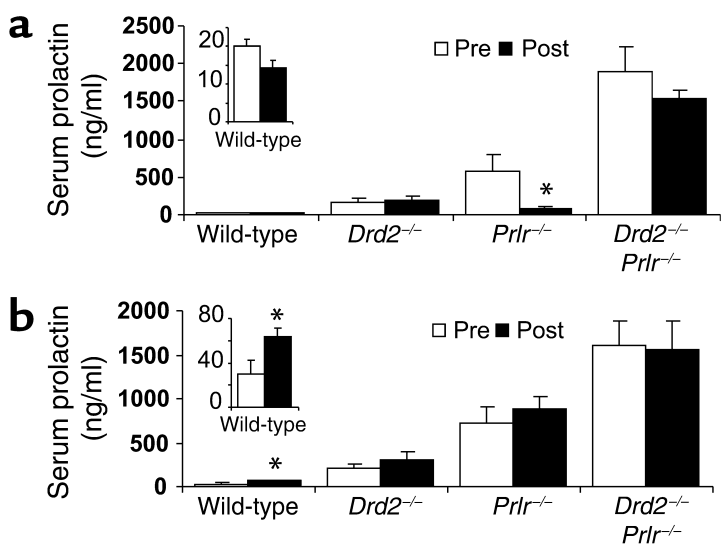

\section{Figure 3}

Serum PRL responses to a dopamine D2R agonist and antagonist in 15-month-old male mice measured one hour after treatment. (a) Bromocriptine decreased PRL levels in Prlr-/- mice, but not the other mutant genotypes. (b) Haloperidol increased PRL levels in wild-type mice but in none of the other genotypes. Because of their low basal values, the wild-type responses are shown magnified in the insets. Statistical analyses were by one-tailed paired Student $t$ test for each drug within genotype. ${ }^{*} P<0.05$ compared with pretreatment; $n=5-8$. 

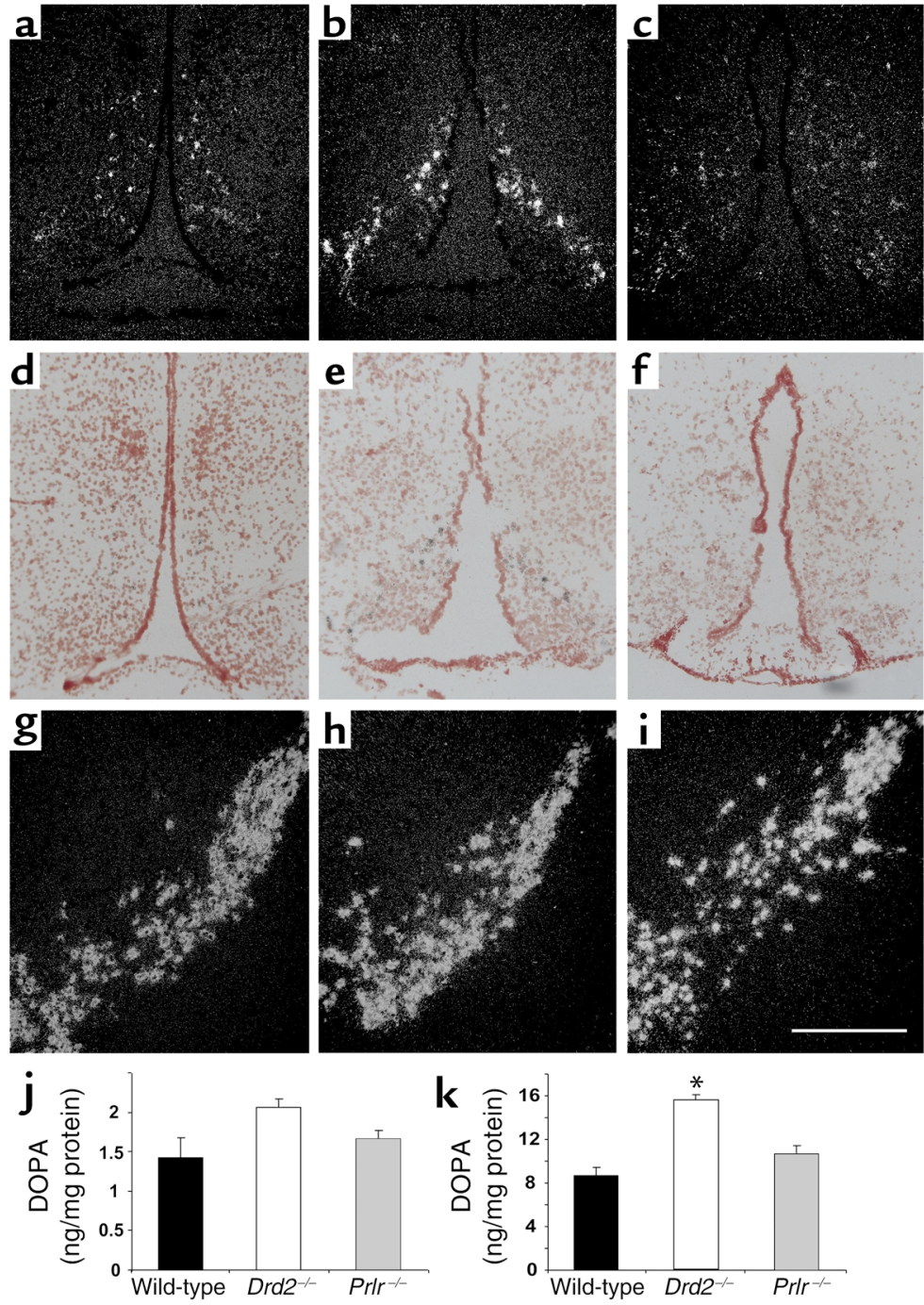

Figure 4

TH expression and activity. (a-i) In situ hybridization for TH mRNA; dark-field (a-c and $\mathbf{g}-\mathbf{i})$ and bright-field (d-f) microscopy. (a and d) Wild-type male mice express TH mRNA in the arcuate nucleus. TH mRNA is increased in $\operatorname{Drd} 2^{-/-}$mice (b and $\mathbf{e}$ ) and appears to be decreased, though clearly still evident, in $\mathrm{PrIr}^{-1-}$ mice (c and $\mathbf{f}$ ). Note comparable levels of TH mRNA in the ventral tegmental area and substantia nigra among the three genotypes: wild type (g), Drd2 ${ }^{--}-(\mathbf{h})$, and $\mathrm{Prlr}^{-/-}$(i). Two or three mice per group were evaluated. (j) Hypothalamic and (k) striatal content of DOPA after NSD 1015 treatment. Striatal DOPA is significantly increased, and hypothalamic DOPA shows a similar trend in Drd2 $2^{-/-}$mice, consistent with increased TH activity in dopamine neuronal terminals. DOPA levels did not differ between $\mathrm{PrIr}^{-1-}$ and wildtype mice in either brain area. Scale bar represents 250 $\mu \mathrm{m}$. Comparison was by ANOVA and Fisher post hoc test. ${ }^{*} P<0.05$ compared with both other groups; $n=4-7$.

absence of inhibitory D2R autoreceptors from the mesostriatal dopamine neurons. However, DOPA levels also tended to be higher in the whole hypothalamus of Drd2 $2^{-/-}$mice. Because the A12 tuberoinfundibular dopamine neurons do not normally express dopamine autoreceptors (38), we believe TH mRNA and DOPA accumulation in the hypothalamus of $\operatorname{Drd2} 2^{-/-}$mice tended to be higher due to increased PRL stimulation of these neurons $(2,5,7)$. Notably, in other models of chronic hyperprolactinemia, exposure of tuberoinfundibular dopamine neurons to very high levels of PRL for extended periods of time abrogates the increase in dopaminergic activity of those neurons and not the survival of dopaminergic neurons in the hypothalamus (36). Consistent with these later data, arcuate TH mRNA levels in the $\mathrm{Prlr}^{-1}$ mice were decreased but still present in a normal distribution in our study and were quantitatively normal in another (37), indicating grossly normal development of hypothalamic dopamine neurons. The reduction in levels of TH mRNA is consistent with PRL's known positive regulatory feedback effects on transcription of the $\mathrm{TH}$ gene. Whether the decreased mRNA levels resulted in decreased amounts of TH enzyme, and whether there were additional changes in TH activity (mediated for example by changes in phosphorylation), was evaluated by measuring DOPA accumulation after blockade of AADC by NSD 1015. An increase in DOPA after NSD 1015 treatment has been shown to correlate with the activity of $\mathrm{TH}$ and with dopaminergic neuronal activity (38). As a control for the pharmacologic treatments, DOPA levels were measured and found to be significantly higher in striata from $D r d 2^{-/-}$mice than in striata from wild-type mice. This finding is consistent with increased $\mathrm{TH}$ activity secondary to the
(39). This may explain why statistically significant changes were not seen in the current study.

In contrast, DOPA accumulation in neither striatum or hypothalamus of $\mathrm{Prlr}^{-1-}$ mice was significantly different from that in the wild type. It is likely that the low baseline levels of dopamine synthesis in male mice in combination with the presence of contaminating nonPRL-regulated catecholaminergic terminals present in the hypothalamic samples precluded detection of any reduction in DOPA accumulation in the hypothalamus of $\mathrm{Prlr}^{-/}$mice. The arcuate nucleus has also been demonstrated to contain a substantial population of neurons that express $\mathrm{TH}$ but not $\mathrm{AADC}$, and therefore normally synthesize L-DOPA as an end product; the accumulation of this L-DOPA would not be directly affected by the inhibition of AADC (40).

Clearly, however, the loss of PRL signaling on hypothalamic dopamine neurons does not entirely explain the phenotypes observed in the two single-knockout mutants. Specifically, hyperprolactinemia has an earlier onset in both sexes of $\mathrm{Prlr}^{--}$mice than in $\mathrm{Drd2} 2^{-/-}$mice and is more severe in $\mathrm{Prlr}^{-/-}$mice than in $\mathrm{Drd2} 2^{-/-}$mice at 
several timepoints. Furthermore, in males there is an additive effect of the compound double knockout $\left(\mathrm{Drd2} 2^{--}, \mathrm{Prlr}^{--}\right)$with regard to both the degree of hyperprolactinemia at various timepoints and the size of pituitary adenoma formation. These differences in phenotype suggest that despite the evidence of reduced dopaminergic input from the experiments discussed above, there must be an additional mechanism for PRL in regulating lactotroph function.

A direct effect of PRL on the pituitary, and possibly even a specific direct effect on lactotrophs, is plausible. PRLRs have been localized to the pituitary gland in several species, including mouse $(10,11)$, but specifically identified on lactotrophs in only two species thus farrat and human $(12,13)$. Our in vitro experiments present evidence of PRL action directly on mouse pituitary cells. Interestingly, PRL was observed to have an inhibitory effect on the proliferation of wild-type female lactotrophs. This effect appears to be completely independent of dopamine, as this in vitro model uses cultured pituitary cells grown in dopamine-free media. In addition, there was a similar inhibitory effect in pituitary cultures from male $\operatorname{Drd} 2^{-/-}$mice. The lack of response in cultures from female $\mathrm{Drd2} 2^{-/-}$mice, but persistent response in cultures from male $\mathrm{Drd2} 2^{-/-}$mice, suggests that the antecedent hyperprolactinemia in the females downregulates the response to further PRL signaling. This is similar to other models of chronic hyperprolactinemia in which there is loss of the normal response of tuberoinfundibular dopaminergic neurons to PRL (39). Indeed, there is evidence that in other tissues PRL regulates its own receptor, with downregulation observed at extremely high concentrations, although there does not appear to be a consistent PRL level at which this downregulation occurs (41-43). The dichotomous response we observed appeared to occur with preceding serum PRL levels of approximately 500 $\mathrm{ng} / \mathrm{ml}$ or greater. This level also represents a cutoff that separates the transition from lactotroph hyperplasia to adenoma in female $\operatorname{Drd2^{-/-}}$ mice $(3,4)$ and distinguishes the microscopic adenomas of male $\operatorname{Drd2^{-/-}}$ mice from the macroadenomas of male $\mathrm{Prlr}^{-1}$ mice and compound mutant $\mathrm{Drd2} 2^{-/}, \mathrm{Prlr}^{-/}$mice.

The inhibitory growth response of lactotrophs to PRL is quite different from the proliferative effect exhibited in nonpituitary cell types (14-19, 21-25), in human prolactinoma cells (44), and in a transformed rat lactotroph cell line (20). It is, however, consistent with models of PRL effects on EGF and TGF- $\alpha$ signaling cascades. Quijano and Sheffield demonstrated that PRL can decrease the growth-promoting action of EGF by increasing threonine phosphorylation and decreasing tyrosine phosphorylation of EGFR (45). Therefore, it may be that PRL exerts an inhibitory effect on lactotroph growth by limiting the actions of growth factors.

It was not possible in our experimental model to determine whether the inhibitory response to PRL was due to a direct action on lactotrophs or instead was mediated by juxtacrine or paracrine involvement of another anterior pituitary cell type. Possible mediation by one or more of the many other modulators of PRL secretion and lactotroph proliferation also cannot be ruled out. Evidence of both autocrine and paracrine regulation of lactotroph growth exists for TGF- $\beta$ (46), EGF (47), galanin (48), and many other factors. Thus, there is abundant evidence for local pituitary control of lactotroph proliferation.

Using the genetic models of $\mathrm{Drd2}^{-/-}$and $\mathrm{Prlr}^{-/-}$mice, we have provided new evidence that PRL is the major physiological regulator of tuberoinfundibular dopamine tone and that PRLR signaling is necessary to maintain functionally relevant levels of dopamine release into the hypophyseal portal-venous circulation. However, the unanticipated phenotypic differences between $\mathrm{Drd2}^{-/-}$and $\mathrm{Prlr}^{-/-}$mice, and the additive or possibly synergistic effects of the two mutations on lactotroph function in male mice, indicate an additional dopamine-independent role for PRL in the inhibition of PRL secretion and lactotroph growth. We demonstrated a profound in vitro inhibitory effect of PRL on lactotroph proliferation in primary mouse pituitary
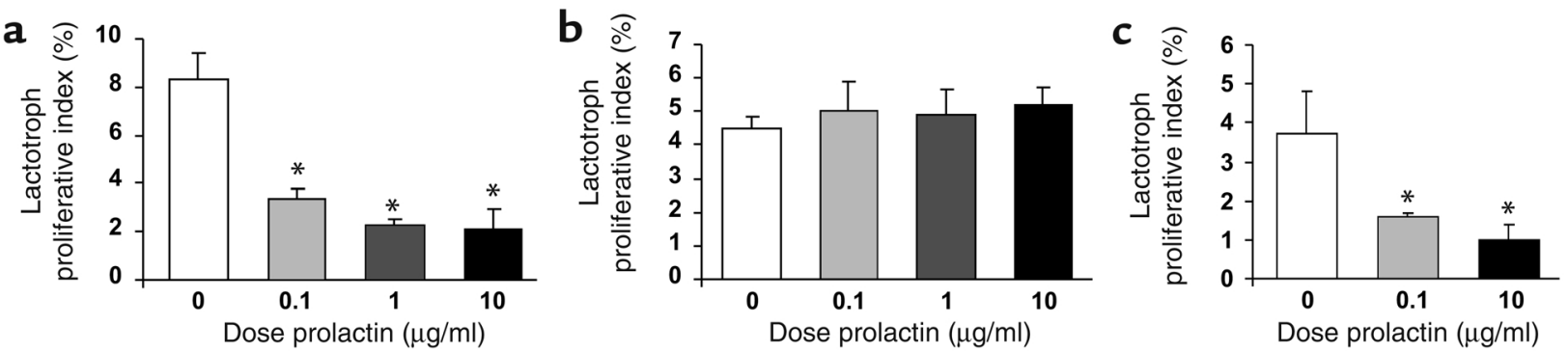

\section{Figure 5}

Effect of PRL treatment on lactotroph proliferation in primary pituitary cultures. Primary cultures were treated with increasing concentrations of $\mathrm{mPRL}$. The proliferative index was calculated as the percentage of cells demonstrating immunoreactivity for both PRL and BrdU relative to the total number of PRL-immunoreactive cells. (a) PRL treatment reduced the proliferative index by a maximum of $75 \%$ in pituitary cultures from wild-type female mice. (b) PRL treatment had no effect on the lactotroph proliferative index in cultures from Drd $2^{-/-}$female mice. (c) PRL treatment reduced the proliferative index by a maximum of $73 \%$ in pituitary cultures from $\operatorname{Drd} 2^{-/-}$male mice. $P<0.001$ for ANOVA of main effect of PRL treatment for wild-type female and $D r d 2^{-/}$male pituitary cultures. ${ }^{*} P<0.001$ compared with control medium without recombinant PRL by Fisher post hoc test. 
cultures, mediated either directly on lactotrophs or by paracrine or juxtacrine mechanisms through other pituitary cells. Growth inhibition was diminished when there was pronounced preceding hyperprolactinemia in vivo, indicating that a loss of growth inhibition by PRL may precede or accompany adenoma formation.

Maximal growth of pituitary tumors and hyperprolactinemia in the mice was observed only when lactotrophs were devoid of both D2R- and PRLR-mediated inhibition. This finding of synergistic effects in male $\mathrm{Drd2}^{-/-}$and $\mathrm{Prlr}^{-/}$mice suggests possible implications for the pathogenesis and management of invasive lactotroph macroadenomas in humans. Furthermore, the finding of such a profound pituitary phenotype of massive lactotroph adenomas in $\mathrm{Prlr}^{-1-}$ mice argues for evaluation of PRLR defects or PRLR signaling defects as a potential etiology of human macroprolactinomas. To date there are very few studies that have analyzed PRLR expression in prolactinomas $(13,49)$, and we are unaware of any studies assessing PRLR binding specifically to the plasma membrane in hyperprolactinemic states. Rather than altering receptor expression, prolonged hyperprolactinemia could alter PRLR signaling; such an evaluation has not yet been performed. Our data indicate the importance of determining specific functions and deficits in PRL signaling under hyperprolactinemic conditions. Such findings could have significant implications for understanding the etiology and progression of these tumors and perhaps for providing additional targets for pharmacologic intervention to complement $\mathrm{D} 2 \mathrm{R}$ agonists.

\section{Acknowledgments}

We thank James Wessel and Kelvin So for technical assistance. This work was supported by NIH grants K08 DK-02477 (K.G. Schuff), T32 DK-07674 (S.T. Hentges), T32 DA-07262 (M.A. Kelly), R01 EY-04864 (P.M. Iuvone), and P01 DK-55819 (M.J. Low), and by a grant from the Institut National de la Santé et de la Recherche Médicale (P.A. Kelly).

1. Asa, S.L., and Ezzat, S. 1998. The cytogenesis and pathogenesis of pituitary adenomas. Endocr. Rev. 19:798-827.

2. Ben-Jonathan, N., and Hnasko, R. 2001. Dopamine as a prolactin (PRL) inhibitor. Endocr. Rev. 22:724-763.

3. Kelly, M.A., et al. 1997. Pituitary hyperplasia and chronic hyperprolactinemia in dopamine D2 receptor-deficient mice. Neuron. 19:103-113.

4. Asa, S.L., Kelly, M.A., Grandy, D.K., and Low, M.J. 1999. Pituitary lactotroph adenomas develop after prolonged lactotroph hyperplasia in dopamine D2 receptor-deficient mice. Endocrinology. 140:5348-5355.

5. Freeman, M.E., Kanyicska, B., Lerant, A., and Nagy, G. 2000. Prolactin: structure, function, and regulation of secretion. Physiol. Rev. 80:1523-1631.

6. DeMaria, J.E., Lerant, A.A., and Freeman, M.E. 1999. Prolactin activates all three populations of hypothalamic neuroendocrine dopaminergic neurons in ovariectomized rats. Brain Res. 837:236-241.

7. Hentschel, K., et al. 2000. Prolactin regulation of tuberoinfundibular dopaminergic neurons: immunoneutralization studies. Brain Res. 852:28-36.

8. Selmanoff, M. 1985. Rapid effects of hyperprolactinemia on basal prolactin secretion and dopamine turnover in the medial and lateral median eminence. Endocrinology. 116:1943-1952.

9. Arbogast, L.A., and Voogt, J.L. 1991. Hyperprolactinemia increases and hypoprolactinemia decreases tyrosine hydroxylase messenger ribonucleic acid levels in the arcuate nuclei, but not the substantia nigra or zona incerta. Endocrinology. 128:997-1005.
10. Tzeng, S.J., and Linzer, D.I.H. 1997. Prolactin receptor expression in the developing mouse embryo. Mol. Reprod. Dev. 48:45-52.

11. Saiardi, A., Bozzi, Y., Baik, J.H., and Borrelli, E. 1997. Antiproliferative role of dopamine: loss of D2 receptors causes hormonal dysfunction and pituitary hyperplasia. Neuron. 19:115-126.

12. Morel, G., Ouhtit, A., and Kelly, P.A. 1994. Prolactin receptor immunoreactivity in rat anterior pituitary. Neuroendocrinology. 59:78-84.

13. Jin, L., et al. 1997. Prolactin receptor messenger ribonucleic acid in normal and neoplastic human pituitary tissues. J. Clin. Endocrinol. Metab. 82:963-968.

14. Vergani, G., Mayerhofer, A., and Bartke, A. 1994. Acute effects of rat growth hormone $(\mathrm{GH})$, human $\mathrm{GH}$ and prolactin on proliferating rat liver cells in vitro: a study of mitotic behaviour and ultrastructural alterations. Tissue Cell. 26:457-465.

15. Dombrowicz, D., Sente, B., Reiter, E., Closset, J., and Hennen, G. 1996. Pituitary control of proliferation and differentiation of Leydig cells and their putative precursors in immature hypophysectomized rat testis. J. Androl. 17:639-650.

16. Boyles-Walsh, E., Shenkin, A., White, M.C., and Fraser, W.D. 1995. Effect of glycoprotein and protein hormones on human meningioma cell proliferation in vitro. J. Endocrinol. 145:155-161.

17. Matsuda, M., Mori, T., Park, M.K., Yanaihara, N., and Kawashima, S. 1994. Enhanced cell proliferation by hyperprolactinemia in both exocrine and endocrine pancreas in mice. Eur. J. Endocrinol. 130:187-194.

18. De Mello-Coelho, V., Savino, W., Postel-Vinay, M.C., and Dardenne, M. 1998. Role of prolactin and growth hormone on thymus physiology. Dev. Immunol. 6:317-323.

19. Cheng, Y., Zhizhin, I., Perlman, R.L., and Mangoura, D. 2000. Prolactininduced cell proliferation in PC12 cells depends on JNK but not ERK activation. J. Biol. Chem. 275:23326-23332.

20. Krown, K.A., Wang, Y.F., Ho, T.W., Kelly, P.A., and Walker, A.M. 1992. Prolactin isoform 2 as an autocrine growth factor for GH3 cells. Endocrinology. 131:595-602.

21. Lee, R.C., Walters, J.A., Reyland, M.E., and Anderson, S.M. 1999. Constitutive activation of the prolactin receptor results in the induction of growth factor-independent proliferation and constitutive activation of signaling molecules. J. Biol. Chem. 274:10024-10034.

22. Mangoura, D., Pelletiere, C., Leung, S., Sakellaridis, N., and Wang, D.X. 2000. Prolactin concurrently activates src-PLD and JAK/Stat signaling pathways to induce proliferation while promoting differentiation in embryonic astrocytes. Int. J. Dev. Neurosci. 18:693-704.

23. Das, R., and Vonderhaar, B.K. 1996. Involvement of SHC, GRB2, SOS and RAS in prolactin signal transduction in mammary epithelial cells. Oncogene. 13:1139-1145.

24. Friedrichsen, B.N., Galsgaard, E.D., Nielsen, J.H., and Moldrup, A. 2001. Growth hormone- and prolactin-induced proliferation of insulinoma cells, INS-1, depends on activation of STAT5 (signal transducer and activator of transcription 5). Mol. Endocrinol. 15:136-148.

25. Lebrun, J.J., Ali, S., Sofer, L., Ullrich, A., and Kelly, P.A. 1994. Prolactininduced proliferation of $\mathrm{Nb} 2$ cells involves tyrosine phosphorylation of the prolactin receptor and its associated tyrosine kinase JAK2. J. Biol. Chem. 269:14021-14026.

26. Kiya, T., et al. 1998. Apoptosis and PCNA expression induced by prolactin in structural involution of the rat corpus luteum. J. Endocrinol. Invest. 21:276-283.

27. Bignon, C., Daniel, N., Kermabon, A.Y., and Djiane, J. 1995. Prolactin induces growth inhibition and promotes differentiation of CHO cells stably transfected with prolactin receptor complementary DNA. FEBS Lett. 358:84-88.

28. Glasow, A., et al. 1998. Differential expression of prolactin receptor (PRLR) in normal and tumorous adrenal tissues: separation of cellular endocrine compartments by laser capture microdissection (LCM). Endocr. Res. 24:857-862.

29. Martini, J.F., et al. 2000. The antiangiogenic factor 16K PRL induces programmed cell death in endothelial cells by caspase activation. Mol. Endocrinol. 14:1536-1549.

30. Ormandy, C.J., et al. 1997. Null mutation of the prolactin receptor gene produces multiple reproductive defects in the mouse. Genes Dev. 11:167-178.

31. Ormandy, C.J., Binart, N., and Kelly, P.A. 1997. Mammary gland development in prolactin receptor knockout mice. J. Mammary Gland Biol. Neoplasia. 2:355-364.

32. Lucas, B.K., Ormandy, C.J., Binart, N., Bridges, R.S., and Kelly, P.A. 1998. Null mutation of the prolactin receptor gene produces a defect in maternal behavior. Endocrinology. 139:4102-4107.

33. Nir, I., Haque, R., and Iuvone, P.M. 2000. Diurnal metabolism of dopamine in dystrophic retinas of homozygous and heterozygous retinal degeneration slow (rds) mice. Brain Res. 884:13-22.

34. Pastorcic, M., De, A., Boyadjieva, N., Vale, W., and Sarkar, D.K. 1995. Reduction in the expression and action of transforming growth factor beta 1 on lactotropes during estrogen-induced tumorigenesis in the anterior pituitary. Cancer Res. 55:4892-4898. 
35. Phelps, C.J., Romero, M.I., and Hurley, D.L. 1995. Role of prolactin in developmental differentiation of hypophysiotropic tuberoinfundibular dopaminergic neurons. Recent Prog. Horm. Res. 50:471-481.

36. Phelps, C.J., and Horseman, N.D. 2000. Prolactin gene disruption does not compromise differentiation of tuberoinfundibular dopaminergic neurons. Neuroendocrinology. 72:2-10.

37. Phelps, C.J., Estrada, I.J., Evdemon-Hogan, M., Binart, N., and Kelly, P.A. 2002. Hypothalamic dopaminergic neurons in prolactin receptor-null mice. In Program and abstracts: The Endocrine Society's 84th Annual Meeting. The Endocrine Society Press. Bethesda, Maryland, USA. 192-193. (Abstr.)

38. Moore, K.E., and Lookingland, K.J. 1995. Dopaminergic neuronal systems in the hypothalamus. In Psychopharmacology: the fourth generation of progress. F.E. Bloom and D.J. Kupfer, editors. Raven Press Ltd. New York, New York, USA. 245-256.

39. MohanKumar, P.S., MohanKumar, S.M.J., Kaleem Quadri, S., and Voogt, J.L. 1997. Chronic hyperprolactinemia and changes in dopamine neurons. Brain Res. Bull. 42:435-441.

40. Ershov, P.V., Ugrumov, M.V., Calas, A., Krieger, M., and Thibault, J. 2002. Differentiation of tyrosine hydroxylase-synthesizing and/or aromatic L-amino acid decarboxylase-synthesizing neurons in the rat mediobasal hypothalamus: quantitative double-immunofluorescence study. J. Comp. Neurol. 446:114-122.

41. Bowen, J.M., Telleria, C.M., Towns, R., and Keyes, P.L. 2000. Downregulation of long-form prolactin receptor mRNA during prolactin-induced luteal regression. Eur. J. Endocrinol. 143:285-292.
42. Barash, I., Cromlish, W., and Posner, B.I. 1988. Prolactin (PRL) receptor induction in cultured rat hepatocytes: dual regulation by PRL and growth hormone. Endocrinology. 122:1151-1158.

43. Rosa, A.A., Ferland, L.H., Djiane, J., Houdebine, L.M., and Kelly, P.A. 1985. Maintenance of prolactin (PRL) binding sites in rat liver cells in suspension culture: effect of PRL and of inhibitors of various cellular functions. Endocrinology. 116:1288-1294.

44. Hosojima, H., and Wyche, J.H. 1985. Prolactin control of growth and prolactin autoregulation in cultured human pituitary cells. Horm. Res. 21:240-245.

45. Quijano, V.J., and Sheffield, L.G. 1998. Prolactin decreases epidermal growth factor receptor kinase activity via a phosphorylation-dependent mechanism. J. Biol. Chem. 273:1200-1207.

46. Hentges, S., Pastorcic, M., De, A., Boyadjieva, N., and Sarkar, D. 2000 Opposing action of two TGF- $\beta$ isoforms on pituitary lactotropic cell proliferation. Endocrinology. 141:1528-1535.

47. Felix, R., Meza, U., and Cota, G. 1995. Induction of classical lactotropes by epidermal growth factor in rat pituitary cell cultures. Endocrinology. 136:939-946.

48. Hammond, P.J., et al. 1996. Signalling pathways mediating secretory and mitogenic responses to galanin and pituitary adenylate cyclase-activating polypeptide in the $235-1$ clonal rat lactotroph cell line. J. Neuroendocrinol. 8:457-464.

49. Ciccarelli, E., et al. 1995. Prolactin receptors in human pituitary adenomas. Clin. Endocrinol. 42:487-491. 